\title{
Research on Application of Logistics Process Reengineering in a Bearing Manufacturing Enterprise
}

\author{
QingqingLu \\ School of Management Engineering, Zhengzhou University, Science Avenue, Zhengzhou, China \\ 957478616@qq.com
}

Keywords: business process reengineering, organizational structure, logistics process

\begin{abstract}
The purpose of this paper is to optimize the logistics process of a bearing manufacturing enterprise and improve customer satisfaction. Which focuses on the application of enterprise process reengineering theory and the bearing manufacturing enterprise is insufficient in the logistics process and problems. First find the relevant points of process reengineering, draw the current business flow chart, and then combine the modern market environment conditions, New and business processes for business. The results show that the restructured business processes can better adapt to the development of enterprise logistics.
\end{abstract}

\section{Introduction}

In terms of the economic situation and market environment of the years, the market environment is facing intensified competition situation and the growing trend of global economic integration, and gradually formed a glob-al market competition environment (QingbingYu,2017).Enterprises face diversification of demand, high technology, border fuzzy and many other problems to be solved. Business for the competition, customers and changing the environment and many other factors, still facing a very grim situation. Enterprise product life cycle is shortening and information technology innovation, urging enterprises to keep up with the pace of the times to accelerate the pace of change. Enterprises in order to adapt to the rapid changes in the market environment, and long-term survival and development is not only need a variety of advanced technical support, but also to quickly change with the modern mode of operation does not match the management of the same time to establish a rapid response to the organizational structure And management mechanisms to respond to changing market conditions. Objectively speaking, this requires the enterprises or between enterprises to speed up the pace of process reengineering.

At present, China's manufacturing-oriented enterprises of the larger scale, broad prospects for development, such as the domestic bearing manufacturing enterprises have a good development scale and prospects. However, the current domestic bearing enterprises in terms of development, in the international development is still in the early stages of the bearing industry there is a broader space for development and a longer way to go, only have been looking forward, not self-proclaimed, not To meet the current situation of thinking and implementation of the "going out" for the international development strategy, will continue to grow in order to have a broader market. 


\section{Literature review and research significance}

\subsection{Overview of research}

At the same time, China's research on BPR, especially tools and methodology has also made great progress include:(Anquan zou,Zhixue Liu,Ying Liu,2008)Aiming at the problems existing in the traditional purchasing logistics process of an iron and steel enterprise, the ABC method was used to reengineer the purchasing logistics process of the enterprise, and the performance evaluation and analysis of the lead time of purchasing, the flexibility of the supply chain, the robustness and the cost of purchasing were carried out.. (Zhen Qin 2009)focuses on the implementation strategy and specific measures of enterprise logistics process reengineering based on the concept of green logistics. (Wei Wang,2013)In order to improve the enterprise's ability to adapt to market changes, increase its own adaptability, reduce costs and shorten production cycle, enterprises must reengineer the logistics system process. (Xi Zhang,2013)Based on BPR business process reengineering theory, this paper analyzes the status quo and problems of power logistics core business process, points out the key factors of implementing BPR in power logistics, and puts forward the countermeasures and measures of visual power logistics core business process reengineering based on BPR.

With the gradual improvement of China's modernization level, people pay more attention to environmental protection and sustainable development. (Li Liu,2017)The penetration of green logistics concept has a certain impact on enterprise development and process reengineering. Under the guidance of this concept, enterprises can reduce the cost of environmental pollution and governance, and promote the long-term development of enterprises.The connotation of BPR is to take the process as the center, release the pressure of traditional theory of organizational division of labor, change the organizational process by guiding customers, adapt to the changes of internal and external environment quickly based on information technology, and achieve the best goal. (Huiqin Qian,2016) analyze the current situation of logistics management in chain retail enterprises and expound the business process reengineering.

\subsection{Purpose and significance of research}

In this paper, the bearing manufacturing process as the research focus, to a manufacturing enterprise as the background, the manufacturing process reengineering theory in-depth study to explore the effect of the theory. The company just after the acquisition soon, the implementation of its process reengineering theory, you can adjust the product structure, reduce agency costs, improve the corporate brand structure system, reduce manufacturing enterprises internal friction. Through the implementation of process reengineering theory, enterprises can establish a more adaptable to the company's development model, the operating mechanism of enterprises more fluid, focusing on the improvement of operating efficiency. The implementation of the theory is also conducive to improving the overall quality of corporate personnel and can urge the expansion of human resources, and gradually form a business as a whole open, staff with the responsibility to play, work proactive and meet customer requirements and customer service purposes Are good corporate culture environment.

\section{An Analysis of the Present Situation of a Manufacturing Enterprise}

\subsection{Company profile}

The bearing manufacturing company mainly engaged in rolling bearing basic theory, product design, process equipment, precision instruments and other integrated technology research and 
development, and assume high-speed precision bearings, special bearings and CNC machine tools, such as special spindle products for small batch trial production and scale Production. Its mature technology and high-tech bearing products are also widely used in medical, metallurgical, automotive, machine tools, office automation and other industries.

\subsection{Corporate Strategic Planning}

In order to be able to better adapt to the growing needs of the international competitive environment, in innovation and development. The overall strategic planning of the technology is to adhere to the market-oriented customer-oriented, innovation as the driving force to coordinate and optimize the organizational structure as the focus, speed up the progress of science and technology, and constantly improve the modern enterprise management system, give full play to tangible assets Performance, improve competitiveness, the development of existing assets of the organization and other advantages. In addition to fully tap the enterprise's intangible assets, optimize the allocation of enterprise resources, multi-angle all-round management, and continuously forge ahead, and gradually formed a prominent business, both independent intellectual property rights, the core competitiveness of enterprises, and achieve high-speed sustainable development of. The overall strategic planning of the enterprise is the implementation of the integration strategy. The basic steps in strategic planning are shown in Figure 1.

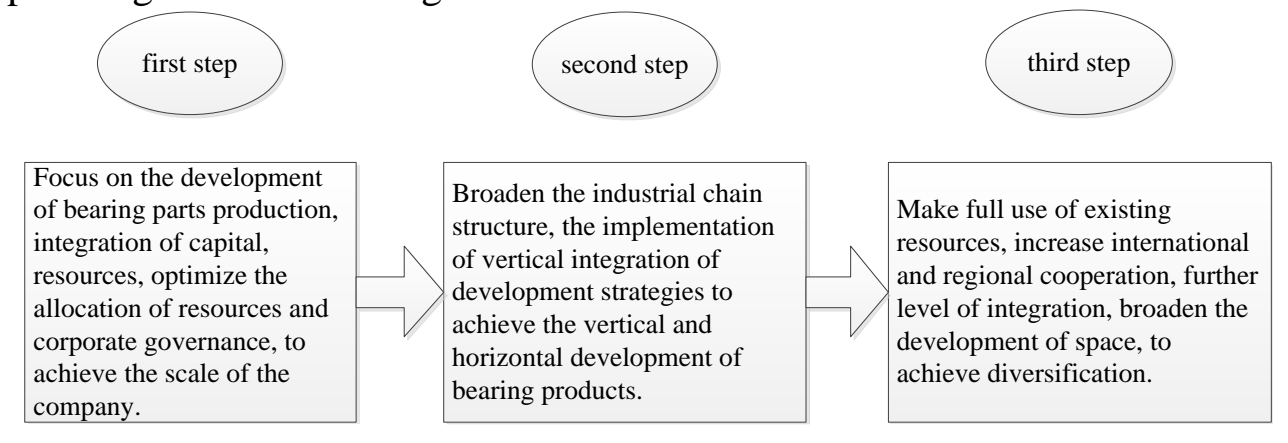

Figure 1 Strategic planning steps.

\subsection{Analysis of the status quo and problems of enterprises}

In this paper, mergers and acquisitions completed soon. Enterprises have a large gap in orders, production costs difficult to control, inventory information are not allowed, the order is mainly multi-species, small quantities, difficult to organize production and inefficient issues such as urgent need to be resolved. In order to fully understand the problems in the enterprise, the internal staff of the inquiry interview implementation of business process reengineering enterprises to adapt to the new model to solve the current problems.

The basic structure of the company is to understand the basis of understanding the enterprise. The organizational model of this paper is shown in Figure 2. The organization structure of the decentralization of serious, including the precision roller bearing manufacturing, the major bearing manufacturing and large bearing manufacturing and heat treatment of the sub-departments have a production department, sales and technical department. This is undoubtedly caused by the management of the organization chaos, there is no unified coordination and management. Such as the production departments under various departments, enterprises in order to arrange production must be coordinated with the production departments of various departments, although the organizational structure of such arrangements contribute to the orderly production, but for the entire enterprise is undoubtedly increased management costs, And coordination of the relevant 
departments consume a lot of time, an increase of time costs to reduce the operating efficiency of enterprises. At the same time for the enterprise purchasing department, it may be because a production department did not coordinate the delay in procurement and thus affect the production planning of other departments. Business costs increase, the benefits will be reduced, is not conducive to long-term development of enterprises. Under the various departments have a sales department, the departments to fight for the performance of the sector and the company appears within the competitive sales market, is not conducive to the sustainable development of enterprises as a group organization. Finally, there is no good communication between departments or departments and business processes, lack of information exchange, which results in slower response to information. Once the views are different, the work efficiency will drop rapidly. Not conducive to sustained and rapid development of enterprises.

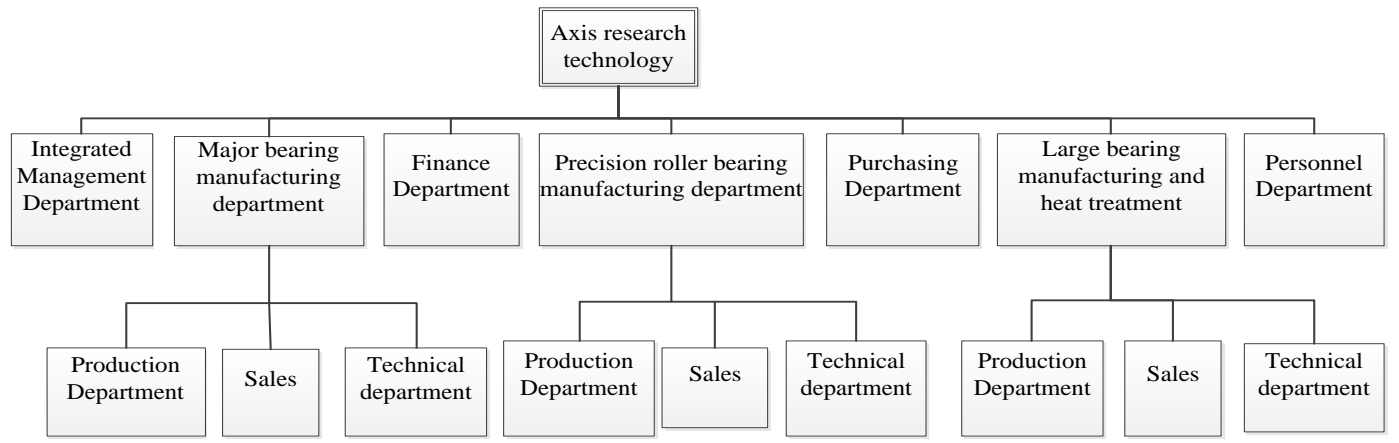

Figure 2 Part of the organization of the organization.

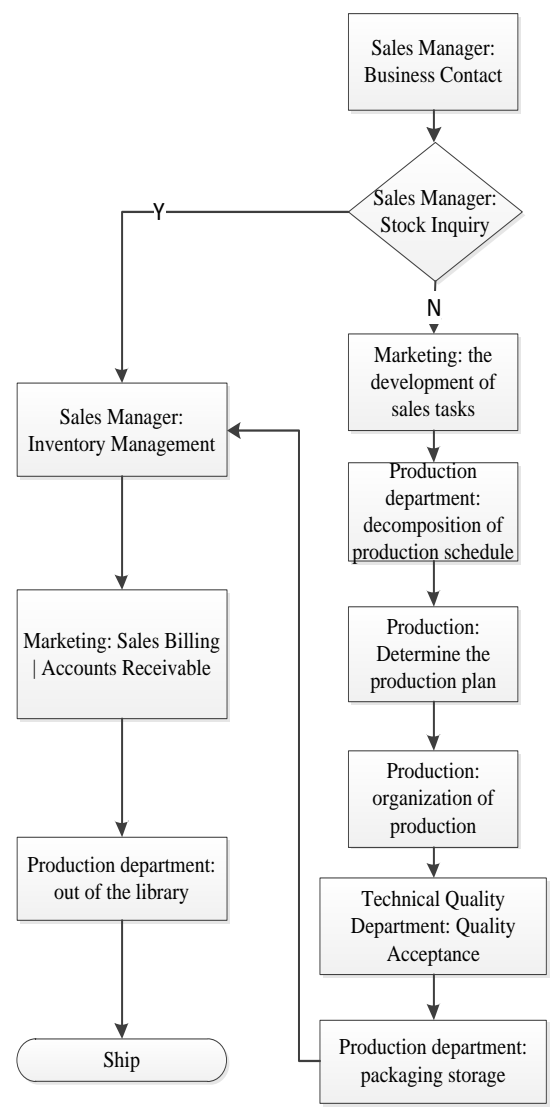

Figure 3 Rebuild the front part of the logistics flow chart. 
Part of the logistics process before the re-engineering as shown in Figure 3. The process is the first customer contact with the company's sales manager, and then through the marketing department, production and other activities after a series of delivery. This process is only blindly down the production, the lack of upward return is feedback information. Easy to cause the production arrangement is unreasonable, the production process is blocked. Only the quality inspection process in the process, did not specify the whereabouts of substandard products, product quality is not guaranteed. Some processes there is no normative phenomenon, or even the lack of part of the process. And the process is the end of delivery, the customer is satisfied with no information channels to understand, is not conducive to business and effective long-term cooperation with customers. Enterprises sometimes exist inventory information is not allowed, but also due to lack of feedback information

\section{BPR implementation method}

\subsection{Implementation strategies}

In the past, the emphasis on improving the enterprise is based on the original basis of the enterprise to make changes to find high efficiency. And business processes highlight the emphasis is a comprehensive attack, re-build, is a change beyond the past, is the original break and then reblend. Therefore, once implemented correctly, its performance is very impressive. The implementation strategy of the program is mainly carried out from the following aspects:

1) Reintegrate existing business processes. Will be the original decentralized work, to re-examine and analyze the process after the process is too long compression, discomfort restructuring, redundant merger.

2) So that employees have the real power of business decisions. Employees need to have a certain range of business decision-making power, do not need every thing to consult the relevant department heads or other departments related to leadership. Employees work together to complete a complete job, take responsibility and quickly make decisions to perform tasks, and optimize service quality. So that employees have a sense of ownership and because of the increase in self-decision-making power, employees in the work of satisfaction has also been significantly improved.

3) Business process diversification. In the process of recycling strategy can be classified according to the nature of the work of the nature of the work in accordance with the nature of the design and processing of the corresponding business processes, when new work, it should be the first classification and select the appropriate business process, so not only Can reduce the complexity of individual processes, but also for the operation of the whole process is more simple and can reduce the working hours.

4) Improve the efficiency of information transmission. In the current situation of the rapid development of information network technology, the use of information network technology can greatly improve the efficiency of information transmission, reduce the consumption of time, improve the competitiveness of enterprises.

\subsection{Methods of the program}

Enterprises through mergers and acquisitions, access to new production technology, manufacturing processes, management and management model. Mergers and acquisitions of the two companies in their organizational structure, core production capacity, enterprise system development, product production processes, human resources, corporate culture, there are differences in the integration of. Mergers and acquisitions of the enterprise process reengineering, 
can make both sides have the advantage to give full play. After the acquisition of enterprises in the business processes and financial processes to re-design, the overall organization of the organizational framework for a thorough transformation of the enterprise's human resources to fully integrate the enterprise system design more innovative, and the two companies Culture to the ultimate blend, which re-build a new corporate culture. In this paper, the company's research technology is facing this situation, mergers and acquisitions completed soon. You can use improved recycling methods, enterprises through mutual integration, to retain the advantages of their own enterprises to abandon their own disadvantages to establish a better enterprise and customer organization and business processes.

\subsection{Implementation of the program}

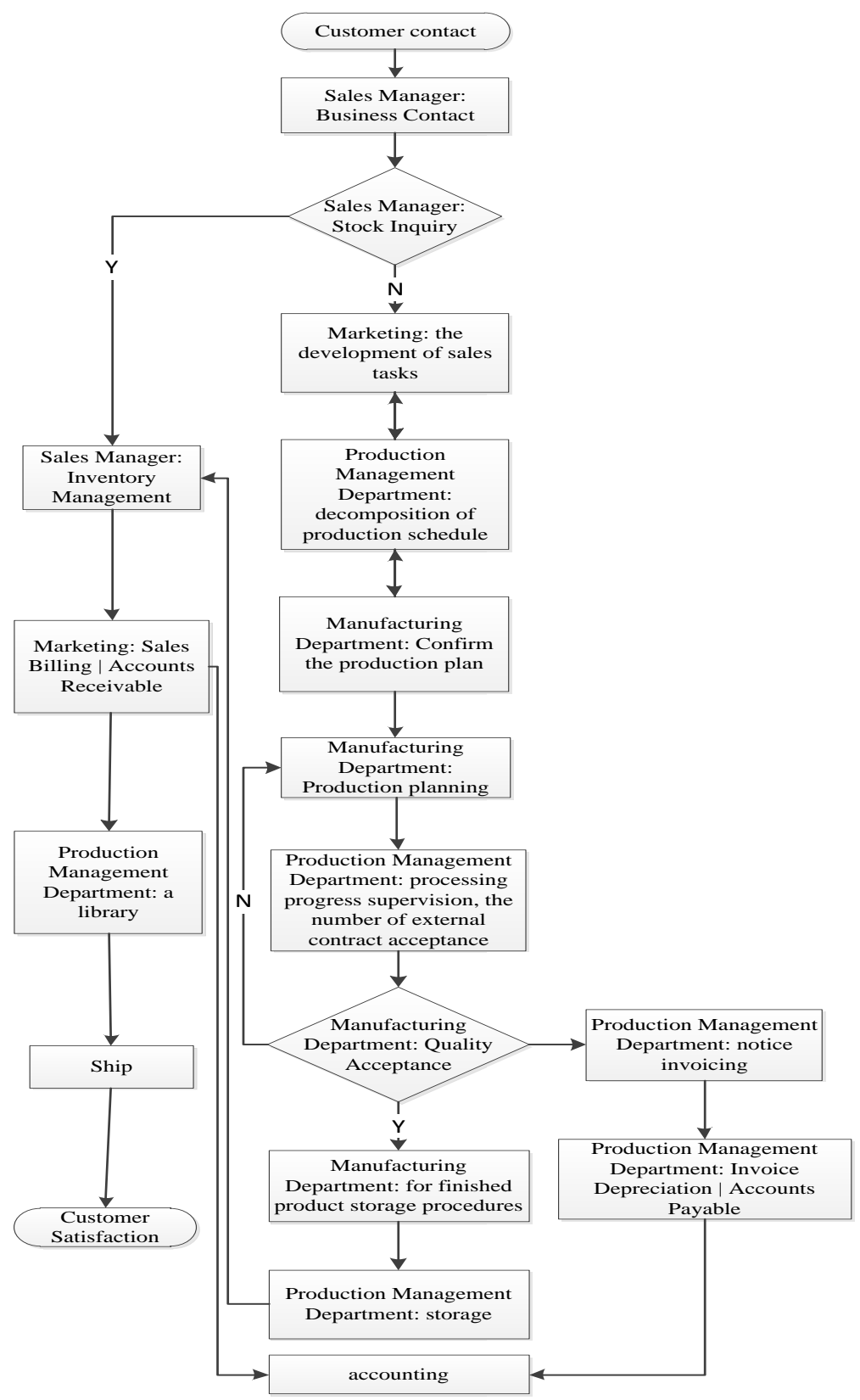

Figure 4 Business process after recycling. 
For the business process after the reengineering, to overcome the process of the existence of the current process of feedback or lack of feedback. Technical marketing and production management, production management and bearing manufacturing, bearing manufacturing and bearing manufacturing have formed between the information feedback mechanism. Marketing Department to develop sales tasks and then by the production management part of the production plan after the production by the Ministry of Bearing to confirm the production plan, began production. The information sharing between departments, reasonable arrangements for production, to achieve the best, to avoid waste. Bearing manufacturing parts processing and production of finished parts are also interconnected. After the recycling process not only has the quality inspection process, but also pointed out the inspection of substandard products, the need to return to the manufacturing department to re-planning production, to ensure quality. The process after the reorganization of the process of regulating some of the process, eliminating the non-standard process. The final process is customer satisfaction as the end of the process. Taking full account of customer satisfaction as the ultimate goal of the process of recycling. See Figure 4 for details.

\section{Conclusion}

Based on the comprehensive analysis of business process reengineering (BPR) theory, this paper reconsidulates the business process of the enterprise and draws out the business flow chart which is more in line with the enterprise status quo. After the reorganization of the production process smoothly. In the production of enterprises, in strict accordance with the plan developed by the budget and estimated costs, procurement management. Operating, to reduce the use of corporate funds to reduce inventory to prevent backlog of products, adhere to the use of zero inventory management. Management, more simple, direct. Improve the organizational structure, so that the organizational structure more adapt to the operation of business processes. Reduce process run time and save time costs. Enterprise organization structure is more rational, enterprises can better meet customer needs. Organize information in a timely manner and form a good communication mechanism. More rational allocation of organizational members of the work, the establishment of a more stable work order, the overall competitiveness of enterprises increased.

\section{References}

[1] Anquan, Zou, Zhixue, Liu, Ying, Liu, 2008. Purchasing Logistics Process Reengineering and Case Analysi-s of Iron and Steel Enterprises.Industrial Engineering. (02), pp.76-81.

[2] Huiqin, Qian, 2016. Logistics management and business process reengineering of chain retailers, Business Economics Research.(11),pp.86-88.

[3] Li, Liu, 2017. Business process reengineering based on green logistics concept. Era finance,(02),pp. 183.

[4] Qingbing, Yu, 2017. Based on the analysis of quality control measures in bearing manufacturing process.Innovation and application of science and technology. (09), pp. 115.

[5] Wei, Wang, 2013. Analysis of process reengineering of enterprise logistics system [J]. Logistics engineering and management,35 (04),pp.33-34.

[6] Xi, Zhang, 2013. Research on BPR-based visual power logistics core business process reengineering. Logistics technology,32 (05),pp.109-111.

[7] Zhen Qin, 2009. Analysis of Enterprise Logistics Process Reengineering Based on Green Logistics Concept [J].Reform and Strategy, 25(05),pp. 173-175. 